\section{ralud mental}

Sánchez Solís, Alejandro; Saucedo Ramos, Claudia Lucy; García Silberman, Sara Análisis de la aproximación de Alcohólicos Anónimos a los pacientes hospitalizados Salud Mental, vol. 38, núm. 3, mayo-junio, 2015, pp. 159-166 Instituto Nacional de Psiquiatría Ramón de la Fuente Muñiz Distrito Federal, México
Salud Mental

ISSN: 0185-3325

perezrh@imp.edu.mx

Instituto Nacional de Psiquiatría Ramón

de la Fuente Muñiz

México

Disponible en: http://www.redalyc.org/articulo.oa?id=58239891002

- Cómo citar el artículo

Número completo

- Más información del artículo

Página de la revista en redalyc.org

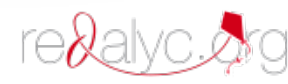

Sistema de Información Científica

Red de Revistas Científicas de América Latina, el Caribe, España y Portugal Proyecto académico sin fines de lucro, desarrollado bajo la iniciativa de acceso abierto 


\title{
Análisis de la aproximación de Alcohólicos Anónimos a los pacientes hospitalizados
}

\author{
Alejandro Sánchez Solís, ${ }^{1}$ Claudia Lucy Saucedo Ramos, ${ }^{2}$ Sara García Silberman ${ }^{3}$
}

Artículo original

\begin{abstract}
Background

The percentage of alcohol dependents who attend self-help groups increased from $33.4 \%$ in 2008 to $43.7 \%$ in 2011 according to the National Surveys of Addictions. Alcoholics Anonymous (AA) and their Twelve Steps program represent the main model for self-help in Mexico. The $12^{\text {th }}$ Step or carrying the message is a common strategy to attract new members into AA groups and it is done frequently at hospital wards.
\end{abstract}

\section{Objective}

To describe the activity of carrying the message in a hospital context in order to identify the meanings involved and how AA members relate to the patients.

\section{Method}

An ethnographic approach was used to observe the activity of nine AA's members in a third level hospital in Mexico City over a ten-month period. The activity system model was used for data organization and interpretation.

\section{Results}

The carrying the message activity consists of six core moments: 1. Self-introduction of the recovering alcoholic; 2. Defining alcoholism as an incurable disease; 3 . AA's Twelve Steps as an alternative; 4. Self-diagnosis; 5. Ask about consumption; 6. Invitation to hospital $A A^{\prime}$ 's group meetings. The $A A^{\prime}$ 's member uses personal experience's stories to convey AA vision about alcoholism and recovering.

\section{Discussion and conclusion}

The carrying the message activity is mainly oriented by the meaning of alcoholism-denial. The AA's actions are designed to avoid denial and to promote disease acceptance. Furthermore, the hospital setting itself legitimizes the AA presence and their Twelve Steps. Carrying the message presents a strategy to diminish stigma related to alcohol dependency which is one of the main obstacles for seeking help.

Key words: Alcohol dependence, hospitals, Alcoholics Anonymous, carrying the message.

\section{RESUMEN}

\section{Antecedentes}

El porcentaje de dependientes alcohólicos que acude a los grupos de autoayuda aumentó del $33.4 \%$ en 2008 al $43.7 \%$ en 2011 según las Encuestas Nacionales de Adicciones. Alcohólicos Anónimos (AA) y su programa de Doce Pasos representa el principal modelo de autoayuda en México. El $12^{\circ}$ Paso o llevar el mensaje es una estrategia común para atraer nuevos miembros a los grupos de AA y se realiza frecuentemente en salas hospitalarias.

\section{Objetivo}

Describir la actividad de llevar el mensaje en un contexto hospitalario para identificar los significados implicados y la manera de relacionarse con los pacientes.

\section{Método}

Se utilizó una aproximación etnográfica para observar la actividad de nueve miembros de $\mathrm{AA}$ en un hospital de tercer nivel de la Ciudad de México a lo largo de diez meses. Se aplicó el modelo del sistema de actividad para la organización e interpretación de los datos.

\section{Resultados}

La actividad de llevar el mensaje tiene seis momentos principales: 1. Autopresentación del alcohólico en recuperación; 2. Definición del alcoholismo como una enfermedad sin cura; 3. Los Doce Pasos de AA como una alternativa; 4. Autodiagnóstico; 5 . Preguntar sobre el consumo; 6. Invitación a las juntas del grupo de AA en el hospital. Los miembros de AA utilizan sus relatos personales para comunicar la visión de AA sobre el alcoholismo y la recuperación.

\section{Discusión y conclusión}

Llevar el mensaje se orienta principalmente por el significado de la negación del alcoholismo. Las acciones de los AA están diseñadas para evitar la negación y promover la aceptación de la enfermedad. Además, el mismo contexto hospitalario legitima la presencia de AA y sus Doce Pasos. Llevar el mensaje nos presenta una estrategia para disminuir el estigma asociado con la dependencia alcohólica, el cual es uno de los principales obstáculos para solicitar ayuda.

Palabras clave: Dependencia alcohólica, hospital, Alcohólicos Anónimos, llevar el mensaje.

Instituto de Ciencias de la Salud de la Universidad Veracruzana.

Facultad de Estudios Superiores "Iztacala" Universidad Nacional Autónoma de México.

Dirección de Investigaciones Epidemiológicas y Psicosociales, Instituto Nacional de Psiquiatría Ramón de la Fuente Muñíz.

Correspondencia: Alejandro Sánchez Solís. Cerrada Texcalatlaco 4, San Miguel Xicalco, Tlalpan, 14490, México DF. Tel: (044-55) 3081-9901. E-mail: sansol@comunidad.unam.mx

Recibido primera versión: 21 de febrero de 2013. Segunda versión: 25 de abril de 2014. Aceptado: 8 de agosto de 2014. 


\section{ANTECEDENTES}

De acuerdo con la Encuesta Nacional de Adicciones (ENA) del 2011, el 6.2\% de la población mexicana entre 12 y 65 años presenta dependencia alcohólica. Además, se ha detectado que sólo una baja proporción de la población consumo-dependiente hace uso de diversos servicios de tratamiento. En la ENA del 2008 se estimó que el 7.6\% de la población dependiente había recibido algún tipo de tratamiento, mientras que en 2011 se calculó a la baja con un 6.8\%; es decir, casi 331 mil de 4.9 millones de personas con dependencia. ${ }^{1}$

Con respecto al tipo de ayuda o tratamiento que busca la población dependiente, hay variaciones a lo largo del tiempo. A finales del siglo pasado se señalaba a los grupos de Alcohólicos Anónimos (AA) como el recurso más utilizado, incluso por encima de los servicios públicos de atención a la salud. ${ }^{2,3}$ Sin embargo, esta preferencia no siempre ha quedado registrada en las encuestas. Por ejemplo, en la Encuesta Nacional sobre Epidemiología Psiquiátrica se observó que los dependientes al alcohol prefirieron acudir a los profesionales de la salud, específicamente a los psiquiatras, más que a los grupos de autoayuda. ${ }^{4}$ En las ENA 2008 y 2011 también se observó una preferencia por acudir a un profesionista, ${ }^{*} 44.5 \%$ y $44.7 \%$ respectivamente. ${ }^{1}$ Sin embargo, hubo un incremento en la opción de autoayuda: del 33.4\% en 2008 al 43.7\% en el 2011. Entre las mujeres, el aumento fue del doble: del 15\% al $31.2 \%$, mientras que en los hombres, la autoayuda se convirtió en la primera opción para el 2011.

Los grupos tradicionales de AA son los representantes principales de la autoayuda o ayuda mutua contra el alcoholismo en México. La asociación Central Mexicana de Servicios Generales de AA (Central Mexicana) contaba con 14600 grupos en el 2012, ${ }^{5}$ mientras que la asociación Corporación de Servicios Tradicionales de AA (Sección México) tiene registrados 2400 grupos. $^{6}$

La vía principal por la cual una persona empieza a participar en un grupo de AA en México es porque un miembro de AA lo invita, lo cual sucede en el $61 \%$ de las ocasiones. ${ }^{7}$ $\mathrm{Al}$ proceso de invitar a un nuevo participante dentro de un grupo de AA se le conoce como $12^{\circ}$ Paso o llevar el mensaje, lo cual consiste en que un miembro de AA narra a otra persona consumidora la experiencia que ha tenido con el alcoholismo y cómo, al practicar los Doce Pasos, logró mantenerse sobrio. ${ }^{8}$ En el 2012 se estimó que el 40\% de los AA llevaron el mensaje en el mes anterior a la encuesta.?

En la última década surgió una línea de investigación sobre las diversas actividades de ayuda mutua que despliega la comunidad de AA tanto al interior como al exterior de la misma. Por ejemplo, llevar el mensaje y el impacto en la abstinencia que alcanzan quienes practican dichas actividades. ${ }^{9}$ En general se observa que, entre más se ayuda a otros alco-

\footnotetext{
* Incluye tratamiento parcial y completo con un profesionista.
}

hólicos, mayor es la correlación con el aumento en el número de días en abstinencia. Así, aunque se señala la necesidad de mayor investigación, los resultados preliminares apoyan la idea de que el $12^{\circ}$ Paso es una actividad que contribuye a la recuperación personal.

Por otro lado, llevar el mensaje también es un mecanismo para el crecimiento de la comunidad desde su origen en 1935. En un estudio internacional que analiza los procesos de adaptación cultural de AA como un movimiento social, Eisenbach-Stangl et al. identificaron diversas formas de AA para vincularse con otras organizaciones como parte de una estrategia para atraer nuevos miembros. ${ }^{10}$ Se observó que la comunidad de AA en México tenía un nivel alto de colaboración con las instituciones de salud, centros de tratamiento en adicciones y prisiones, permitiéndoles acceder a la población dependiente cautiva. Así, el 71\% de los AA mexicanos habían llevado el mensaje, siendo el segundo porcentaje más alto entre los países participantes. ${ }^{11}$

En particular, las salas hospitalarias representan un contexto óptimo para llevar el mensaje. Entre 2004 y 2012 se registraron 130 mil egresos por enfermedades atribuibles al alcohol en los hospitales del sector salud. ${ }^{12}$ Es por esto que la comunidad de AA establece y renueva sus convenios de cooperación con las instituciones de salud y centros de tratamiento para tener acceso a esta población. ${ }^{5}$

Hoy en día, la Central Mexicana de AA colabora con el Instituto Mexicano del Seguro Social (IMSS) para el tratamiento y rehabilitación de derechohabientes con problemas de alcoholismo. ${ }^{13}$ Por otro lado, en el Instituto de Seguridad y Servicios Sociales de los Trabajadores del Estado (ISSSTE) las actividades de detección oportuna del personal de salud son complementadas con la transmisión del mensaje de AA en las salas de hospitalización. ${ }^{14}$ Por último, se lleva a cabo la "Semana Nacional del Enfermo Alcohólico Encamado" en conjunto con la Secretaría de Salud, desde $2009 .{ }^{15}$

A pesar de la presencia de AA en los hospitales, son pocas las investigaciones que analizan cómo se concreta la actividad de llevar el mensaje. Por ejemplo, Collins et al. sólo describen las maneras de colaboración existentes entre AA y hospitales y el impacto potencial en la comunidad, ${ }^{16}$ mientras que Blondell et al. evaluaron el efecto de llevar el mensaje de AA en pacientes hospitalizados por traumatismos relacionados al consumo de alcohol. ${ }^{17}$ Estos últimos autores observaron que una intervención breve por parte de un médico, seguida de la visita de un AA, fue la condición experimental que se correlacionaba con el mayor número de días de abstinencia y de pacientes que buscaron tratamiento o participaron en un grupo de ayuda mutua al año de seguimiento.

Blondell et al. describen brevemente la intervención de AA como una visita de 30-60 minutos realizada en pares. Los AA buscaban pacientes con características semejantes (sexo y raza), no daban consejos ni hacían recomendaciones sobre el tratamiento, sólo se limitaban a compartir sus historias personales y su "experiencia, fuerza y esperanza". 
Dado que AA tiene fuerte presencia en las instituciones de salud en nuestro país, y en función de que no contamos con información detallada sobre sus acciones al interior de las salas hospitalarias, el objetivo de este artículo es ofrecer una descripción analítica del método de aproximación de los AA cuando están frente a los pacientes hospitalizados. Los resultados forman parte de una investigación más amplia que tuvo como objetivos describir la estructura organizacional de AA, sus estrategias para acceder y permanecer en contextos hospitalarios, así como analizar las acciones y los significados de llevar el mensaje que dan sentido al proceso de recuperación de los propios miembros de AA. ${ }^{18}$

\section{MÉTODO}

Se utilizó un método etnográfico para observar, registrar y analizar la actividad de llevar el mensaje por parte de los AA en un contexto hospitalario y entender qué significados le atribuían a dicha actividad desde sus propias experiencias. En un sentido amplio, la etnografía supone analizar y entender el sentido y significado de las acciones que las personas tienen en sus contextos socioculturales. Se trata de una aproximación a la cotidianeidad de las personas en su medio que requiere de observaciones analíticas sistemáticas, entrevistas a profundidad, análisis de textos y archivos necesarios. ${ }^{19}$ El etnógrafo busca entender la actividad humana y respeta las construcciones culturales que observa; además, se esfuerza por conocer a profundidad las maneras de comportarse de las personas y el lazo que los une tanto en el encuentro cara a cara como en las estructuras sociales que organizan su actuar. La etnografía nos permitió entender y analizar cómo la comunidad de AA participaba en un contexto hospitalario y cómo sus miembros que llevaban el mensaje a pacientes hospitalizados lograban significar su propia sobriedad al mismo tiempo que fortalecían la imagen de AA como fundamental en el proceso de atención al alcoholismo. De acuerdo con lo anterior, no se analizaron las reacciones de los pacientes hospitalizados ni su sentir hacia lo que los miembros de AA les compartían, puesto que nuestra intención fue entender a profundidad el sistema de actividad desplegado por la comunidad de AA en los hospitales.

\section{Contexto hospitalario y participantes}

La selección de los participantes se basó en los siguientes criterios: a) quienes tuvieran experiencia en el hecho de llevar el mensaje; b) que fueran participantes diversos: expertos, novatos, diferentes años de participación en AA; y c) antigüedad en la colaboración existente entre AA y los hospitales.

El hospital seleccionado corresponde al tercer nivel, en el cual se brinda atención médica general y especializada a población abierta. Está ubicado en la zona centro del Distrito Federal y ha colaborado con AA desde la década de 1970; en el área de hospitalización se reúne un grupo de AA tres veces por semana.

Nueve miembros de AA aceptaron ser observados durante sus recorridos al llevar el mensaje. El rango de edad osciló entre 25 y 65 años. Cinco de ellos contaban con ocho o menos años en AA; uno tenía 14 años y tres llevaban más de 20 años. Con respecto a los años llevando el mensaje, tres de ellos tenían menos de un año, otros tres, entre dos y tres, y tres más, seis años. Los datos específicos se presentan en el cuadro 1.

\section{Trabajo de campo}

El periodo de acceso al campo transcurrió entre enero y abril del 2008 y se caracterizó por un proceso de familiarización con la organización y el lenguaje de AA. Posteriormente, entre julio del 2008 y mayo del 2009 se realizaron las observaciones directas de llevar el mensaje. Durante el desarrollo de la actividad no se hicieron registros con la finalidad de respetar a los pacientes y a los propios miembros de AA, de manera que las notas de campo se elaboraron al salir de las salas de hospitalización. En ocasiones se entregaron copias de los registros a los miembros de AA para su lectura y verificación.

\section{Sistema de actividad}

Se utilizó el modelo del sistema de actividad propuesto por Engeström ${ }^{20}$ como la herramienta analítica para organizar los datos de las notas de campo en las siete categorías teóricas que, según dicho autor, constituyen la actividad humana: sujeto, objeto, instrumentos, comunidad, reglas, división del trabajo y resultados. En el análisis destacamos cómo los miembros de AA utilizaban diversos instrumentos materiales y culturales (folletos, discursos, historias, registros) para dar cuerpo al sistema de actividad de llevar el mensaje ante los pacientes hospitalizados. Por último, se analiza el sistema desde dos perspectivas: la perspectiva del sujeto de la actividad y la perspectiva externa al sistema. Con la primera se elaboró una representación de llevar el mensaje con base

Cuadro 1. Edad, años en la comunidad y años llevando el mensaje de los nueve miembros observados durante la actividad.

\begin{tabular}{|c|c|c|c|}
\hline Miembros de AA & Edad & Años en $A A$ & $\begin{array}{c}\text { Años llevando } \\
\text { el mensaje }\end{array}$ \\
\hline 1 & 40 & 5 & $<1$ \\
\hline 2 & 45 & 6 & $<1$ \\
\hline 3 & 25 & 4 & $<1$ \\
\hline 4 & 36 & 8 & 2 \\
\hline 5 & 48 & 7 & 2 \\
\hline 6 & 47 & 14 & 3 \\
\hline 7 & 52 & 21 & 6 \\
\hline 8 & 63 & 21 & 13 \\
\hline 9 & 65 & 28 & 14 \\
\hline
\end{tabular}


en las acciones individuales de los AA, mientras que la segunda nos permitió analizar aspectos de la relación entre la comunidad de AA y el contexto hospitalario. Engeström ${ }^{19}$ propone utilizar ambas perspectivas para generar un análisis dialéctico entre las perspectivas subjetiva y sistémica.

\section{Consideraciones éticas}

Los participantes voluntarios fueron informados sobre el propósito de la investigación, garantizando su anonimato y la confidencialidad en el uso de la información. Se solicitó verbalmente su consentimiento para ser acompañados y observados durante sus recorridos por las salas del hospital.

\section{RESULTADOS}

La comunidad de AA tiene varias décadas llevando el mensaje en el hospital en el que se realizó la investigación, por lo tanto, existen días y horarios establecidos para los recorridos en determinados pabellones. Cerca del 30\% de los encuentros eran visitas de seguimiento, ${ }^{*}$ es decir, visitas a pacientes previamente identificados como posibles alcohólicos por otros pacientes o por el personal de salud, principalmente por enfermeras. La forma más común de aproximarse era de uno a uno, y en menor frecuencia los AA iban en parejas. Los encuentros tenían una duración promedio de ocho minutos y, cuando se identificaba a un posible alcohólico, se extendían el doble de tiempo. Existían diferencias en la forma de llevar el mensaje por parte de los miembros de AA, ya que algunos hablaban más de su pasado alcohólico, mientras otros preferían hablar de su recuperación y de los beneficios de AA. Por otro lado, entre ellos había distintos estilos para presentarse: amables, solemnes o con humor. También existían diferencias entre los novatos y los más experimentados, ya que los primeros eran más formales, se preocupaban por seguir un orden en la presentación durante sus recorridos, en tanto que los veteranos eran más espontáneos, flexibles, con mayor seguridad en su forma de hablar y mayor expresión de sus emociones. No obstante, ya fueran novatos o veteranos, la actividad de llevar el mensaje transcurría en seis momentos definidos: 1. Presentación, 2. Definición del alcoholismo, 3. AA como una alternativa, 4. Autodiagnóstico, 5. Preguntar sobre el consumo y 6. Invitación a participar en un grupo. A continuación exponemos cada momento.

\section{Soy un alcohólico en recuperación}

$\mathrm{Al}$ aproximarse a los pacientes, los AA se presentan como alcohólicos en recuperación. Hacen explícito su agradeci-

\footnotetext{
* El proceso de seguimiento en $\mathrm{AA}$ no requiere que sea siempre el mismo compañero quien visite al paciente en varias ocasiones. Se recomienda que sean varios compañeros, para ofrecer más experiencias de recuperación al posible paciente alcohólico.
}

miento hacia el personal de salud por permitirles estar ahí para ofrecer información sobre alcoholismo y para ayudar a otros alcohólicos, por ejemplo cuando expresan:

"Venimos de AA. Agradecemos a los médicos que nos dejen estar aquí y que nos dejen compartir nuestras experiencias." Veterano, 13 años llevando el mensaje.

"Las autoridades del hospital nos permiten venir, porque ellos también se preocupan por esta terrible enfermedad." Novato, 2 años llevando el mensaje.

Durante la actividad de llevar el mensaje, los AA ponían esmero en su arreglo personal y comentaron que al presentarse aseados y ordenados en su vestimenta combatían las ideas comunes sobre las personas alcohólicas. Así, afirmaron que incluso los propios consumidores tenían la idea de que los alcohólicos son borrachos de la calle o "teporochos". La actividad de llevar el mensaje está tan estructurada y planeada que incluso la atención al arreglo personal es un detalle que se cuida para no fomentar las creencias adversas al proceso de recuperación.

\section{El alcoholismo es una enfermedad, no un vicio}

Al hablar del alcoholismo con los pacientes siempre lo proponían como una enfermedad. Los miembros de AA leían o parafraseaban definiciones sobre alcoholismo hechas por otras organizaciones, siendo la más común la propuesta por la Organización Mundial de la Salud en 1952: "El alcoholismo es una enfermedad de carácter físico, psíquico y social, que además es progresiva y mortal". A esta definición le añadían una característica: la falta de voluntad para controlar el consumo de alcohol. Los AA explicaban que el alcoholismo era como un tipo de "alergia", porque sólo bastaba un trago de alcohol para generar una obsesión y compulsión por la bebida.

La mayoría de los AA recordaba y compartía con los pacientes, cómo ellos mismos reaccionaron cuando se les habló del alcoholismo como una enfermedad. En general, describían que sintieron un gran alivio cuando se les explicó que un alcohólico no puede controlar el consumo de alcohol. Algunos enfatizaban que al aceptar su enfermedad dejaron de luchar contra sí mismos y de culparse por no poder beber de manera contenida, como mucha gente lo puede hacer.

También mencionaban que una de las características principales del alcoholismo era la negación de la propia enfermedad. Nuevamente, al hablar de la negación, lo hacían por medio de los relatos personales. Por ejemplo, uno de los AA veteranos explicaba que, a pesar de las consecuencias que sufría, no veía el origen en el consumo de alcohol:

"La enfermedad del alcoholismo me hizo sufrir mucho. Perdí trabajo, dinero, amigos y familia. Pasó mucho tiempo para que pudiera entender que tenía una enfermedad. Golpes, problemas de dinero, discusiones, malestares. Cuando bebía, sentía que todos estaban en mi contra y querían hacerme enojar. Me tardé en reaccionar y en aceptar". Veterano, 14 años. 
Advertían que cualquier persona podría llegar a ser alcohólica sin importar edad, sexo, educación o condición socioeconómica. Por último, señalaban que hasta ahora no se conocía ningún tratamiento o medicina que curara al alcoholismo o que devolviera al alcohólico el control para beber sin problemas.

Esta manera de posicionar al alcohólico y sus circunstancias de vida les permitía ofrecer un marco moral a los pacientes para que no se sintieran desvalorizados, sino comprendidos y con posibilidades de cambio.

\section{Los grupos de AA como una solución}

Después de ofrecerles relatos sobre sus propias vidas, acerca de cómo ellos mismos lograron aceptar que tenían un problema de salud, empezaban a hablar del programa de los Doce Pasos de los grupos de AA. No hacían una descripción de los Pasos, sino que hablaban de la vida en los grupos y de cómo se compartían experiencias e ideas que ayudaban para dejar el alcohol. Algunos contaban sus primeras impresiones cuando llegaron a un grupo:

"Cuando un alcohólico quiere dejar de beber, en AA puede encontrar la manera. Los grupos y los compañeros apoyan para que el alcohólico pueda alcanzar la sobriedad". Novato, menos de un año.

"Sólo en los grupos pude detener el consumo y entender mi problema de raíz (...) Me costó mucho trabajo entender que no podía controlar mi manera de tomar alcohol". Veterano, 13 años.

Para los miembros de AA era importante aclarar que los grupos no son religiosos ni son centros de reclusión o anexos. Dado que existen organizaciones religiosas dedicadas a la rehabilitación de las adicciones que venden artesanías o comida como una forma de obtener recursos, los AA se desligaban de ellas. También marcaban la diferencia con respecto a los centros de internamiento que utilizan el programa de los Doce Pasos* y enfatizaban que la gente no reconoce la diferencia entre los grupos de AA y los anexos, lo que provoca mala fama y que se crea que en ambos existe maltrato:

"No amarramos, no castigamos o encerramos. Existen otros que pueden hacer eso, pero no AA. Se trabaja de manera voluntaria, no cobramos por lo que hacemos ni esperamos donativos de la gente. Lo único que queremos es transmitir información valiosa sobre el alcoholismo". Veterano, seis años.

Así, en pasajes breves, los AA exponían cómo ellos mismos lograron sobriedad al participar en un grupo y cómo no eran lugares de maltrato, con lo cual buscaban convencer implícitamente al paciente hospitalizado.

\section{Autodiagnóstico}

A cada paciente visitado se le regalaba un folleto con información general sobre AA y el alcoholismo. En el folleto tam-

* Aunque no los señalaban explícitamente, se referían a los centros de internamiento llamados "anexos". bién se lee un cuestionario que sirve para saber si alguien es alcohólico, con 12 preguntas sobre las consecuencias del consumo, pérdida de control y pensamientos que justifican la embriaguez. ${ }^{* *}$ En cada pregunta se lee también una reflexión desde la perspectiva de AA. Las preguntas se responden afirmativa o negativamente. Al final, se señala que cuatro o más afirmaciones indican la posibilidad de tener un problema severo.

Informaban que las preguntas debían ser contestadas de manera individual. Al obsequiar los folletos aclaraban que no estaban insinuando que el paciente era alcohólico. Invitaban a los pacientes a regalar el folleto a otra persona en caso de que ellos no tuvieran problemas con el alcohol.

\section{5. ¿Y usted bebe?}

Después de entregar el folleto, se preguntaba por el consumo de alcohol. La forma de preguntar no era agresiva ni incisiva; era pronunciada con tacto. Las respuestas de los pacientes eran variadas, desde ser abstemios o tomar una o dos copas sin problema, hasta los que admitían ser alcohólicos. Aproximadamente una cuarta parte respondió que tenía un tipo de problema o era alcohólico. Los miembros de AA no evaluaban el tipo de problema o el grado de severidad, sólo se enfocaban en el hecho mismo de la aceptación.

A partir de este punto se seguían estrategias distintas. Con aquellos pacientes que decían no tener problemas y que no estaban en seguimiento, se les agradecía su atención y se despedían con palabras de ánimo. Con los pacientes que aceptaban algún problema o su alcoholismo, se les narraban relatos sobre el proceso de la aceptación o la llegada al grupo. Por ejemplo, uno de los veteranos narró a un paciente que había tardado más de diez años en aceptar su alcoholismo, hasta que tocó fondo cuando fue hospitalizado por una enfermedad del hígado. El veterano le señaló lo difícil que era reconocer la enfermedad, al tiempo que lo alentaba al asegurarle que había dado el primer paso.

Por otro lado, a los pacientes que se les creía en estado de negación, se les narraban más relatos personales sobre las consecuencias negativas del alcoholismo (violencia intrafamiliar o pérdida de la esposa e hijos; problemas económicos, laborales o de salud; peleas y problemas con la policía). Un tipo de relato frecuente era la narración del propio paso por el hospital.

"Sé lo que es estar en un hospital, sé lo que se siente estar en una cama de éstas. Yo tuve un accidente y pues estuve grave. Me intervinieron, fue un cirugía fuerte, pero sigo viviendo, aún no me tocaba". Veterano, seis años.

Algunos recurrían a la condición del paciente para relacionarlo con el alcohol y confrontarlo. Por ejemplo, preguntaban si estaban en el hospital por el alcohol, al tiempo

** El autodiagnóstico o cuestionarios puede revisarse desde: http://www. aamexico.org.mx/Tieneproblemas.php. 
que les señalaban una pierna inmovilizada. Otros utilizaban el humor, incluso el sarcasmo con la intención de confrontar las resistencias que el paciente dejaba ver en relación con su alcoholismo. Uno de los novatos hizo referencia a la ocasión en que él mismo recibió el mensaje:

"Ya llevo seis años de sobriedad gracias a este programa. Si no, ya estuviera muerto o en un hospital. ¡Mira!, a mí también me vinieron a pasar el mensaje, porque de veras, igualito que tú, nomás decía que no: ¡Yo no soy alcohólico! ¡Alcohólicos los otros mensos, pero yo no! A mí me dijeron: ¿Qué... te quieres morir ya o qué?" Novato, menos de un año.

Por otro lado, a los pacientes que referían lo difícil que era dejar de beber debido a la influencia de los amigos, se les confrontaba señalando la ausencia de éstos en el hospital:

"¿Y dónde están tus cuates ahora? Yo aquí no veo a nadie. ¿Han venido a verte?"

Si después de dos o tres confrontaciones los pacientes se mantenían diciendo que no tenían problemas con el alcohol, entonces los AA dejaban de insistir. En su lugar, retomaban el tema del cuestionario y agradecían la atención que les ponían. En ese sentido, se mostraban respetuosos con el actuar de los pacientes.

\section{Solicitar datos personales al paciente e invitarlo al grupo}

Quienes tenían problemas o aceptaban su alcoholismo eran invitados a participar voluntariamente en una reunión del grupo institucional de AA en el mismo hospital.* Se les decía que en estas juntas se compartían más experiencias de alcoholismo y de recuperación. Advertían que las juntas eran independientes del tratamiento médico que recibían, pero que era necesario tener la autorización del médico tratante para que el paciente pudiera salir de la sala de hospitalización. El propósito de que asistieran los posibles alcohólicos a una junta era continuar con el proceso de llevar el mensaje y mostrar la dinámica de trabajo en los grupos de AA.

Al final de la actividad, los miembros de AA se mostraban satisfechos aun cuando no hubieran identificado algún paciente alcohólico o uno que pudiera entrar en la lista de seguimiento. En AA se cree que la aceptación del alcoholismo es un proceso largo, por tal razón, muchos pacientes no siempre reconocen su enfermedad. Su satisfacción radica en que han contribuido a acelerar el proceso, ante lo cual comentan "la semilla de AA está echada".

\section{DISCUSIÓN Y CONCLUSIÓN}

La comunidad de AA sabe por tradición que los hospitales son un paso obligado en las trayectorias de vida de muchos

* El grupo institucional se refiere tanto al espacio físico que facilitan las instituciones públicas o privadas ajenas a $\mathrm{AA}$, como a los miembros de $\mathrm{AA}$ que realizan las juntas semanales. dependientes alcohólicos. Con base en esto, AA ha desarrollado un sistema de actividad para promover la aceptación del alcoholismo durante la hospitalización. Llevar el mensaje tiene una lógica similar a la estrategia de salud pública de detección, intervención y referencia a tratamiento en el sentido de que se concibe el paso por el hospital como una oportunidad para promover el cambio. ${ }^{21}$

Con el uso de relatos personales cargados de significados sobre el alcoholismo y los procesos de recuperación, los AA entran en contacto con los pacientes. Estos instrumentos narrativos presentan pasajes de vida que pueden ser interpretados por los pacientes para establecer un sentido de sí mismo y de la relación con el alcohol. En pocas palabras, los AA ofrecen al paciente un marco explicativo sobre su condición de hospitalizado.

Por otro lado, la intencionalidad de la actividad de llevar el mensaje anticipa la negación del alcoholismo; actitud propia de muchos pacientes hospitalizados. La negación previene a los AA sobre qué decir y cómo decirlo; los hace cautelosos. Señalar a un paciente como alcohólico sin que él mismo lo asuma es tenderse una trampa: si éste lo niega, ya no se sabrá si dice la verdad o si es la negación de un alcohólico. Como los propios AA lo señalaron, para evitar avivar la negación del paciente ante su alcoholismo, tenían estrategias muy calculadas para saber hasta dónde insistir en la reflexión sobre las consecuencias en la salud y cuándo detenerse para que el paciente no sintiera que se le estaba forzando. Así, en la actividad de llevar el mensaje uno de los resultados esperados es que los AA sientan que logran sembrar la semilla de la duda sin tensar el encuentro con el paciente.

Los AA se presentan a sí mismos como alcohólicos en recuperación y con ello buscan atraer la atención de los posibles alcohólicos. En AA se cree que los alcohólicos no aceptan su alcoholismo porque les produce culpa, enojo y vergüenza su incapacidad por controlar el consumo. Este razonamiento está asociado a la idea de vicio, la cual remite a la dimensión moral del comportamiento y a la falta de voluntad para poder beber como una persona que sabe contenerse.

En sus relatos, los AA incluyen episodios de hospitalización y sobre la negación del alcoholismo para, de esta manera, darle pauta al paciente y entienda que su condición actual es también una trayectoria de alcohólico. En ese contexto narrativo se significa la hospitalización como señal del alcoholismo y la antesala al grupo de AA. Entonces, las narraciones personales se utilizan como instrumentos para describir el proceso de la aceptación e inicio de la recuperación.

Otra estrategia para mitigar la negación por parte del paciente hospitalizado es el uso del cuestionario para el proceso del autodiagnóstico. El cuestionario presenta una caracterización del alcohólico para promover en los pacientes la identificación personal. En AA se reconoce que pocos alcohólicos aceptan su enfermedad en el primer encuentro, por ello les dejan el cuestionario como una forma de ayudar al paciente a enfocarse en el proceso de la autoaceptación (diferenciándolo 
de la aceptación o confesión frente a otros). De esto modo, llevar el mensaje es una actividad que empata con el proceso lento de la autoaceptación del alcoholismo.

Por último, recurren a los conceptos establecidos por instituciones de salud reconocidas para definir el alcoholismo como una enfermedad; como una condición ajena a la voluntad personal. El argumento del alcoholismo como enfermedad está implícito en la misma presencia de AA en el hospital. Por ejemplo, uno de los AA novatos lo presentó así: "El alcoholismo es una enfermedad, no un vicio. Si no fuera así, entonces ¿por qué nos dejan entrar aquí?". El acceso a las salas del hospital legitima las acciones y concepciones de AA sobre el alcoholismo. Así, con la presentación de sí mismos como alcohólicos y del alcoholismo como enfermedad buscan disminuir la negación y promover la aceptación por parte de los pacientes.

En el contexto médico los pacientes hospitalizados sólo son atendidos en relación con las consecuencias de salud que acarrea el alcoholismo, mientras que AA ofrece alternativas para mantener la sobriedad, con lo cual también busca legitimarse como comunidad. En ese sentido, el contexto hospitalario valida el programa de los Doce Pasos y lo promueve al permitir la presencia de un grupo de AA en el hospital y hasta en el hecho de canalizar pacientes hacia esos grupos.

La relación con el hospital establece un contexto de significación para quienes llevan el mensaje. Se produce un valor por compartir un saber acerca del alcoholismo que los profesionales de la salud no poseen. Así, quienes antes no recibieron ayuda médica para dejar de consumir, ahora apoyan a los médicos para ayudar a otros alcohólicos.

En el presente artículo nos hemos enfocado en el análisis de la actividad de los miembros de AA al llevar el mensaje, y sostenemos que esta actividad tiene implicaciones positivas tanto para los AA en lo individual, que dicen mantenerse sobrios al llevarla a cabo (un aspecto que no ha sido analizado en la línea de investigación sobre conductas de ayuda y abstinencia desarrollada por Zemore et al.), ${ }^{9}$ como para el crecimiento de la comunidad en general. Particularmente, muestra una manera de enfrentar el estigma asociado al alcoholismo, el cual representa un obstáculo para recibir ayuda o iniciar un tratamiento..$^{22,23}$ Concluimos que llevar el mensaje es una actividad muy estructurada que contempla tanto las reglas de participación de la comunidad de AA como los propios estilos que sus miembros logran en la experiencia de participación con los pacientes hospitalizados.

Como limitación de esta investigación debe señalarse la ausencia de datos sobre la población femenil, dado que no se permitió el acceso a las salas de mujeres. Por último, consideramos oportuno señalar como tema para una investigación futura, el efecto de la actividad de llevar el mensaje en los pacientes que lo reciben; es decir, los procesos de motivación al cambio que se generan tras las visitas de los AA en sus circunstancias de hospitalización.

\section{Financiamiento}

Ninguno.

\section{Conflicto de intereses}

Los autores declararon no tener conflicto de intereses.

\section{Agradecimientos}

Para la realización de esta investigación se contó con una beca por parte del Consejo Nacional de Ciencia y Tecnología. También se agradece al grupo institucional de AA que colaboró durante la investigación.

\section{REFERENCIAS}

1. Medina-Mora ME, Villatoro-Velázquez JA, Fleiz-Bautista C, Téllez-Rojo MM et al. Encuesta Nacional de Adicciones 2011: Reporte de alcohol. México: INPRFM, INSP, Secretaría de Salud; 2012.

2. Mariño MC, Medina-Mora ME, Escotto J, De la Fuente JR. Utilización de servicios en una muestra de alcohólicos mexicanos. Salud Mental 1997;20(supl. julio):24-31.

3. Rosovsky H. Alcohólicos Anónimos. En: FISAC (ed.). Beber de tierra generosa. Ciencia de las bebidas alcohólicas. México: FISAC; 1998.

4. Borges G, Medina-Mora ME, Wang P, Lara C et al. Treatment and adequacy of treatment of mental disorders among respondents to the Mexico National Comorbidity Survey. Am J Psychiatry 2006;163(8):1371-1378.

5. Nanni R. El papel de los grupos de ayuda mutua en la atención del alcoholismo en los servicios de salud del primer nivel de atención. En: CONADIC (ed.). Actualidades en adiciones. Prevención y tratamiento adicciones servicios de salud. Libro 2. México: Secretaría de Salud; 2012.

6. Corporación de Servicios Tradicionales de AA. Sección México. Quiénes somos. Recuperado en enero 2013, desde http://www.aa.org. $\mathrm{mx} /$ index.php?option $=$ com_wrapper\&view $=$ wrapper\&Itemid $=9$

7. Central Mexicana de Servicios Generales de AA. Encuesta 2012. Comité de cooperación con la comunidad profesional. Recuperado en Marzo 2014, desde http://www.aamexico.org.mx/CCCP/pdf/Encuesta_2012.pdf

8. Central Mexicana de Servicios Generales de AA. Alcohólicos Anónimos. México; 1986.

9. Zemore SE, Pagano ME. Kickbacks from helping others: Health and recovery. En: Galanter M, Kaskutas L (eds.). Recent Developments in alcoholism. Research on Alcoholics Anonymous and spirituality in addiction recovery. New Jersey: Springer; 2008; pp.141-166.

10. Eisenbach-Stangl I, Rosenqvist P. Variations of Alcoholics Anonymous. En: Eisenbach-Stangl I y Rosenqvist P (eds.). Diversity in unity: Studies of AA in eight societies. Helsinki: Nordic Council for Alcohol and Drug Research Publication; 1998.

11. Mäkelä K, Arminen I, Bloomfield K, Eisenbach-Stangl I et al. Alcoholics Anonymous as a mutual-help movement. A study in eight societies. Madison: University of Wisconsin Press; 1996.

12. Base de datos de egresos hospitalarios por morbilidad en Instituciones Públicas, 2004-2011. México: SINAIS, Secretaría de Salud. Consulta realizada el 31 enero del 2014. Desde: http://www.sinais.salud.gob.mx/ basesdedatos/cubos.html

13. Echevarría S. PrevenIMSS en la prevención universal de las adicciones. En: CONADIC (ed.). Actualidades en adiciones. Prevención y tratamiento adicciones servicios de salud. Libro 2. México: Secretaría de Salud; 2012.

14. Castillo R, Blanco M, De la Rosa B. El problema de las adicciones en el contexto del Instituto de Seguridad Servicios Sociales de las Trabajadores del Estado (ISSSTE). En: CONADIC (ed.). Actualidades en adiciones. Prevención y tratamiento adicciones servicios de salud. Libro 2. México: Secretaría de Salud; 2012. 
15. Central Mexicana de Servicios Generales de AA. Cuaderno de trabajo. $3^{\text {a }}$ Semana nacional del enfermo alcohólico encamado. Recuperado en enero 2013, desde: http://www.aamexico.org.mx/cct/Material/cuadernillo_3a_seaa.pdf

16. Collins GB, Barth J. Using the resources of AA in treating alcoholics in a general hospital. Hosp Community Psychiatry 1979;30:480-82.

17. Blondell RD, Looney SW, Northington AP, Lasch ME et al. Using recovering alcoholics to help hospitalized patients with alcohol problems. J Fam Pract 2001;50(5):447.

18. Sánchez A. "Llevar el mensaje": Análisis de las prácticas de Alcohólicos Anónimos en un contexto hospitalario. Tesis doctoral en psicología. México: Universidad Nacional Autónoma de México; 2013.

19. Hammersley M, Atkinson P. Etnografía. Métodos de investigación. Barcelona: Paidós; 1994.
20. Engeström Y. Activity theory and individual and social transformation. En: Engeström Y, Miettinen R, Punamäki RL (eds.). Perspectives on activity theory. Reino Unido: Cambridge University Press; 1999.

21. Bischof G, Reinhardt S, Freyer-Adam J, Coder B et al. Severity of unhealthy alcohol consumption in medical inpatients and the general population: is the general hospital a suitable place for brief interventions? Int J Public Health 2010;55(6):637-643.

22. Borges G, Medina-Mora ME, Lara C, Zambrano J et al. Alcohol use and alcohol use disorders in Mexico. Contemp Drug Probl 2007;34:389-410.

23. Borges G, Medina-Mora ME, Orozco R, Fleiz C et al. Unmet need for treatment of alcohol and drug use in four cities in Mexico. Salud Mental 2009;32(4):327-333. 